\title{
ABOUT THE SUBDIVISION OF INDOOR SPACES IN INDOORGML
}

\author{
Abdoulaye A. Diakité ${ }^{\text {a,* }}$, Sisi Zlatanova ${ }^{\text {a }}$, Ki-Joune Li ${ }^{\text {b }}$ \\ a 3D GeoInformation, Department of Urbanism, Delft University of Technology, \\ Jaffalaan 9, 2628 BX Delft, The Netherlands - (a.a.diakite, s.zlatanova)@ tudelft.nl \\ b Department of Computer Science and Engineering, Pusan National University, Busan 46241, Korea - lik@ pusan.ac.kr
}

\author{
Commission VI, WG VI/4
}

KEY WORDS: Indoor space, subdivision, IndoorGML, 3D indoor, Navigation graph, IFC, CityGML

\begin{abstract}
:
Boosted by the dynamic urbanization of cities, indoor environments are getting more and more complex in order to be able to host people properly. While most of our time is spent inside buildings, the need of GIS tools to assist our daily activities that can become tedious, such as indoor navigation or facility management, became more and more urgent. In that perspective, the IndoorGML standard is aiming to address the gaps left by other standards regarding the spatial modelling for indoor navigation. It includes several concepts such as the organization of the spaces into cells along with their network representation and the possibility to represent multiple connected layers. However, being at its first stage, several concepts of the standard could be improved. One of these is the cell subspacing that is not enough discussed in the current version of the standard. In this paper, we explore all the aspects involved in the subdivision process, from the identification of the navigable and non-navigable space cells to the generation of a navigation graph. We propose several criteria on which the indoor sub-spacing can rely to be automatically performed and and illustrate them on a 3D indoor model.
\end{abstract}

\section{INTRODUCTION}

The importance of dealing with the indoor environment in GIS is not to be justified anymore. Indeed, early studies in the beginning of the 21th century have shown that people spend most of their time (about $87 \%$ on average) in building's interior (Klepeis et al., 2001). Even though this study was specific to the case of the USA, it is believed to be reflective of the behaviour of most of the urban populations in the world. This observation allowed to attract the attention of the GIS community that used to focus on outdoor features. Furthermore, the worldwide intense urbanization observed since the middle of the 20th century brought also new challenges. According to a study of the United Nations, $66 \%$ of the world's population is projected to be urban by 2050 (Nations, 2014), therefore the buildings will tend to be more and more, high, spacious and complex in order to host all those people while guaranteeing all the safety and well-being conditions.

The growing availability of 3D acquisition tools based on laser or photogrammetry technologies made the modelling or urban environments easier and more accurate. 3D GIS is actively being boosted by those techniques, and the CityGML open standard by OGC (Open Geospatial Consortium, 2012) has been introduced in order to provide adequate support for a proper building and exchange of those massive data. Closer to the building design field where a new paradigm known as Building Information Modelling (BIM) took over, another standard, namely the Industry Foundation Classes (IFC) were also introduced (Building SMART International, 2013). The goal is to allow smoother interoperability in the digital exchanges between actors of a same construction project, but also assist analysis and maintenance of the building during its whole life cycle. While IFC has a deeper focus on indoor than CityGML, both include geometric, topological and semantic classes aiming to model the indoor environment. Never-

* Corresponding author theless, the indoor features (e.g. building components, furnitures, spaces, etc.) as they are currently proposed by those standards are not adapted to applications such as indoor navigation that requires more specific spatial features.

In order to complement the existing standards, IndoorGML (Lee et al., 2014) has been recently adopted by OGC. It provides additional encoding features for indoor spatial information and covers all the necessary requirements to indoor applications such as indoor navigation. With its two main core components, IndoorGML provides classes to describe mainly connectivity relationships and a multi-layered model for the indoor spaces on the one hand and a data model dedicated to navigation on the other hand. The idea is to provide a framework focussed on aspects such as the representation of spatial components (rooms, corridors, etc.) and the localization of indoor features rather than representation of architectural components (Li, 2016). This data model enables applications based on indoor location-based services (LBS).

However, while the current version of IndoorGML thoroughly addresses some of its conceptual aspects such as the cellular notion of spaces as well as their topological, semantic and multilayered representations (Kang and $\mathrm{Li}, 2017$ ), some other aspects lack of clear definition. It is the case for the notion of space subdivision that is considered in the standard but discussed only briefly. Indeed, the complexity of indoor environments populated by objects and people makes it not relevant to consider indoor spaces simply as the spaces bounded by the structural elements, as it used to be with the other standards. By introducing the notion of spatial subdivision, IndoorGML allows more sophisticated implementation of fine-grained indoor applications. Yet, no clear definition has been provided in the characteristics of such subdivision process in the standard.

In this paper, we propose a deeper study of the space subdivision 
concept of IndoorGML. We identify two critical aspects that need to be considered in such process: the occupancy of the indoor space that affects the notion of indoor cells on one hand and the definition of criteria to support the automation of the process on the other hand. We therefore propose:

- an estimation of the real free space available for navigation,

- rules on which an automatic subdivision could be based,

- a study of the node placement in a sub-spaced environment

- and finally the extraction of a navigation graph.

The output of this study could be used for an improvement of the future version of the standard.

In Section 2, we present the researches and developments related to IndoorGML in general and to spatial subdivision in particular. The definition of the indoor space and the consideration of its occupancy will be elaborated in section 3, while in section 4 we will develop all the aspects related to the sub-spacing process. We study the extraction of a navigation graph and its specificities in the context of subdivided spaces before concluding by a discussion at sections 6 .

\section{RELATED WORK}

The IndoorGML standard (Lee et al., 2014, Kang and Li, 2017) is the result of several years of active research targeted to the specific needs of indoor environment. The initiative to make a standard out of it has been undertaken in the last decade ( $\mathrm{Li}$ and Lee, 2010). The idea was to extend the CityGML standard and implement deeper concepts related to the description of indoor spaces (Kim et al., 2013). Therefore there is a strong correlation and interoperability between the two standards as discussed in (Kim et al., 2014) and (Ryoo et al., 2015).

IndoorGML integrates several concepts that are widely recognised in the indoor navigation research community. Therefore, more and more researches and developments are relying on it and tend to produce models following its spatial framework. In that context, (Hwang et al., 2012) developed an editor and a viewer for IndoorGML to mainly support related researches, concerned about representation methods for $2 \mathrm{D}$ and $3 \mathrm{D}$ indoor space and connection method between indoor and outdoor. Semi-automatic methods to generate IndoorGML data from images is presented in (Kim and Lee, 2015). The authors relied on image segmentation and classification methods to identify specific features such as doors and extract the corresponding connectivity graph with indoor spaces such as corridors. Similarly, (Mirvahabi and Abbaspour, 2015) proposed an automatic approach to extract IndoorGML data from OpenStreetMap (Haklay and Weber, 2008). Few researches are also trying to improve some aspects of the current standards, e.g. for specific users such as visually impaired people (Ryu et al., 2014, Iida et al., 2015).

Regarding the specific issue of the subdivision of the indoor spaces, the notion has been just briefly discussed in the official document of the IndoorGML standard. However, several researches have been conducted in that direction (Zlatanova et al., 2014, Khan et al., 2014, Diakité and Zlatanova, 2016). The issue of subdividing the indoor space into functional spaces to allow advanced navigation is developed in (Zlatanova et al., 2013) and (Krūminaite and Zlatanova, 2014). The authors emphasize the importance of a 3D description of the space and the necessity to rely on semantic information for efficient systems. (Becker et al., 2009) introduced a conceptual framework aiming to bridge the gap between representation models for route planning and indoor localization, as the two have different points of interest. The authors proposed a multilayered space-event model to allow both to be performed on a same 3D model. The model can be used to define subdivision of building space according to user profile (walking, driving), security or property regulations, and it is the concept on which the current version of IndoorGML relies. The support of the IndoorGML sub-spacing based on the Land Administration Domain Model (LADM) is also discussed in (Zlatanova et al., 2016b) and (Zlatanova et al., 2016a). Finally, the specific case of the sub-spacing process in IndoorGML is also discussed in (Jung and Lee, 2015), with a focus on spaces of type hall and square and relying on the notions provided in the actual standard.

However, none of those researches directly address the sub-spacing issue in IndoorGML in the context of furnished 3D indoor environment. Indeed most of the works either rely on 2D floor plans and do not consider the indoor environment as populated by objects, while the latter information is critical to precise and finegrained indoor navigation. Therefore, we propose in this paper a deeper study of the problems involved in such space subdivision process and we provide solutions to deal with it in the context of the IndoorGML framework.

\section{CONSIDERING THE STATIC OCCUPANCY OF THE INDOOR SPACE}

IndoorGML has a clear focus on space available for navigation in the indoor environment. However, the standard does not explicitly considers the indoor feature such as furniture that usually occupy the spaces statically. By static occupancy, we refer to indoor objects that imply a physical occupancy of the space while conserving a fixed location in the indoor environment. In this section, we first explore the definition of an indoor space in IndoorGML and we discuss how the occupancy can be considered.

\subsection{The Indoor Space and its Cellular representation in In- doorGML}

In the current version of IndoorGML (v1.02), an indoor space is defined as "a space within one or multiple buildings consisting of architectural components such as entrances, corridors, rooms, doors, and stairs". Thus, IndoorGML considers the entire indoor space as a whole, defined by the notion of cellular space that is composed by a set of the cells forming the indoor space. Additionally, semantic, geometric and topological connectivity information of those cells can also be included in the cellular space. The standard provides categories to classify the cells in a way relevant to indoor navigation issues as well. It is then possible for a cell to be categorized as navigable or non-navigable for example. Figure 1 illustratres all the classes allowing such cell classification.

The IndoorGML's definition of the indoor space fits quite well with the IFC and CityGML standards at the difference that it is fully oriented and focussed on the space, allowing this way to go deeper on related notions (e.g for indoor LBS), where the other standards are limited. This matching between IndoorGML and CityGML or IFC makes it easy to derive IndoorGML classes from them. For example IFC provides a class named IfcSpace 


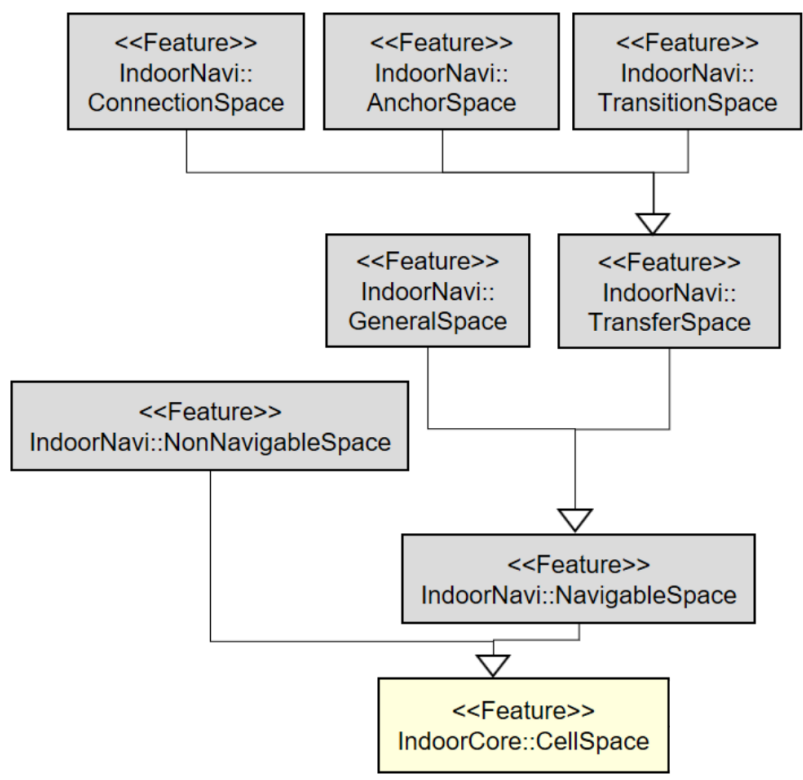

Figure 1. Classes involved in the classification of the space cells (Kang and Li, 2017).

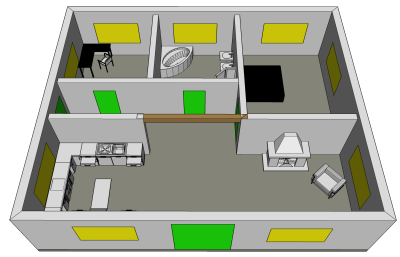

(a)

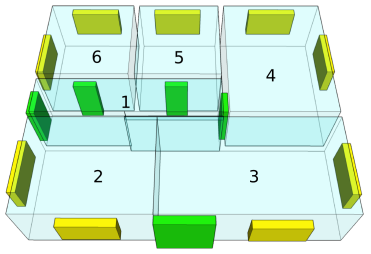

(b)
Figure 2. (a) Furnished IFC 3D model. (b) Original indoor space units in the model.

that could be seen as an indoor cell space. This is illustrated in Fig. 2 with a furnished IFC model and the IfcSpace entities coming along with it.

We can notice here that the space units provided in Fig. 2(b) mostly follow the structural layout of the indoor environment and then encompass the furnishing elements. This means that the space they represent corresponds to the space of the rooms when they are empty. This is another case of good matching between the IFC standard and the IndoorGML one because it is also specified in the latter that IndoorGML is "[...] not concerned about architectural components themselves (e.g. roofs, ceilings, walls) but instead the spaces (e.g. rooms, corridors, stairs) defined by architectural components, where objects can be located and navigate" (Lee et al., 2014). However, there is a fundamental difference that needs to be considered for IndoorGML, as the latter is concerned about spaces available for indoor navigation and cannot assume that space cells are empty, when they are not. Therefore, the furnishing elements cannot be ignored while defining the cellular space.

\subsection{Space cells considering furniture}

As stressed out in (Diakité and Zlatanova, 2016), the features populating the indoor environment have to be considered when it comes to issues related to indoor navigation. Therefore, a direct

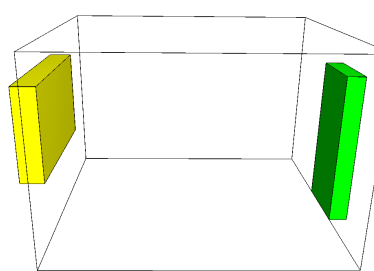

(a)

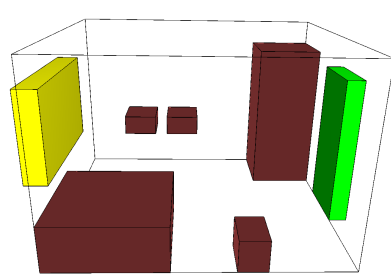

(c)

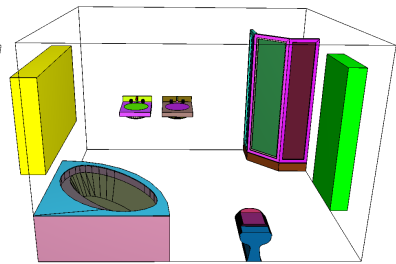

(b)

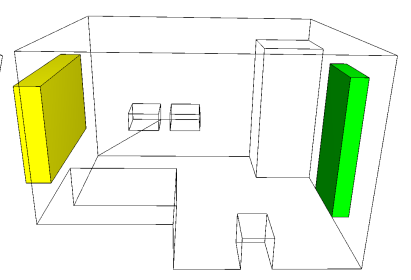

(d)
Figure 3. Difference in the indoor space when considering the furnishing elements. (a) An empty bathroom represented by different volumes: the room layout (wire-frame), a window (yellow) and a door (green). (b) The furnishing objects. (c) Identification of the space occupied by the furnishing elements (dark red). (d) Actual free space in the room.

use of the space units as described in the other standards may not be readily appropriate for some navigation cases. Figure 3 illustrates the difference between considering and ignoring the indoor components in the specific case of the space unit 5 in Fig. 2(b), corresponding to a bathroom. The room as illustrated in Fig. 3(a) is the way that indoor spaces are often represented in such standard. An application based on IndoorGML and using such 3D model would then rely on those spaces, but not all the space they represent is free for navigation, as shown in Fig. 3(d).

The representation shown in Fig. 3 corresponds to what is called a "Thick Door Model" in IndoorGML, meaning that the spaces are represented by solids. The cells in Fig.3(a) are here the room layout, the door and the window volumes. It is then possible to clarify their status in the IndoorGML framework by specifying if the corresponding spaces are navigable, general or transfer spaces, and in the latter case if they are connection, transition or finally anchor spaces. Following that logic, the three volumes in Fig. 3(a) would be categorized this way: the cell corresponding to the room would be a navigable-general space, the door would be a navigable-transfer-connection space and the window could be either like the door or simply non navigable. What appears when relying on the model of Fig. 3(b) is that the classification of the room becomes clearly biased because part of it is non-navigable. Thus, this binary subdivision of the room into occupied and non occupied subspaces, as shown in Fig. 3(c) allows to define an IndoorGML model where the dark-red spaces would be categorized as non-navigable and the rest of the room (see 3(d)) as navigablegeneral space. The full process leading to such binary subdivision is exposed in the work of (Diakité and Zlatanova, 2016).

This distinction between occupied and free space brings already a first sub-spacing level to indoor spaces. Although it does not disturb the structure of the IndoorGML framework, as it fits well with the classification of the occupied cells as non-navigable, it implies the reconsideration of the cells that remains for indoor navigation. It is mainly those cells that were initially targeted in the standard when introducing the notion of subspaces. There- 


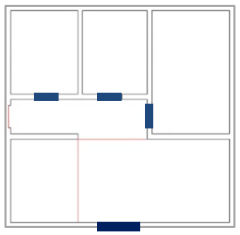

(a) Geometry mode
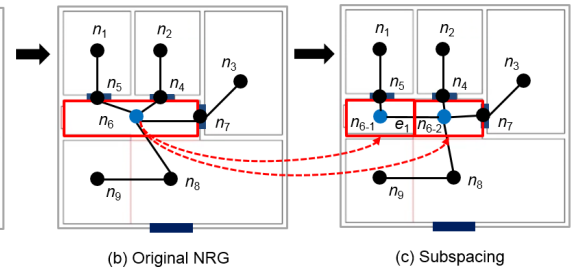

Figure 4. Sub-spacing example discussed in (Lee et al, 2014).

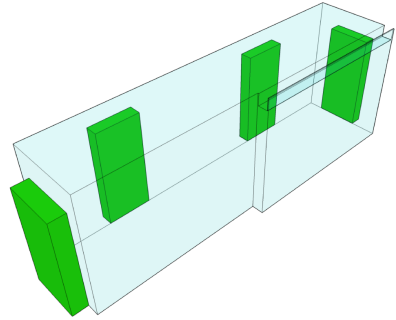

(a)

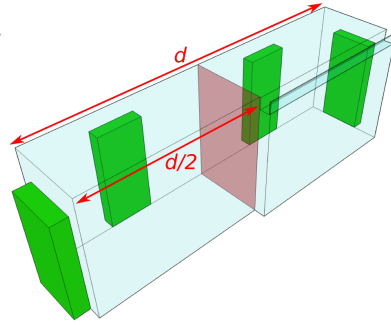

(b) fore, we will now study how to further proceed to such subdivision through several possible criteria.

\section{SUBDIVISION OF SPACE CELLS}

The indoor space has often an hierarchical structure that is different from its architectural structure, independently of its size and complexity. This hierarchical structure results generally from the use that we make of the indoor environment. This can be implicitly implied through the geometric configuration of structural features (e.g. the position of a door, the shape of a corridor, etc.) or through a specific furniture configuration, or the nature and function of the objects in the indoor environment. Because of those different possibilities, an indoor space cannot be seen as one simple and uniform space. IndoorGML being an indoor navigationoriented standard, this means that one simple node may not be enough to conveniently represent a space in the navigation graph.

It is recognised in the standard that "feature such as corridor or hall may be divided to accurately represent the geometric properties of indoor space based on the connectivity relationships among space objects" (Lee et al., 2014). It is also mentioned how indoorGML supports such sub-spacing with the mean of the multilayered space model. Every subdivision granularity can then be considered as a level of the final graph of the model. Figure 4 provides an illustration of such process. Starting from a geometry model, the original node-relational graph (NRG) is built by abstracting each space cell with the mean of a node, and their link through edges, following the Poincaré Duality. It then appears that the cell of the corridor is represented with only one node. Looking at the geometric structure of that space, one central node do not really reflect its characteristic, considering that it is linked to several doors. Therefore a simple sub-spacing is proposed with the virtual cutting of that cell in two subspaces, leading to two nodes in the NRG, instead of one. Nevertheless, there is no direction provided in how such sub-spacing could be automatically performed on a $3 \mathrm{D}$ model.

It can happen that the designer of the original model considers already a spatial subdivision, independently from the architectural structure of the building. This can be observed in Fig. 2, where the rooms 2 and 3, plus the corridor (room 1) spatially corresponding to one architectural space in Fig. 2(a), were already separated in three pieces. This results from the decision of the designer to make a difference between the corridor and the room in one hand, even though there is no door to separate them, and on the other hand to separate the lower part into two subspaces to make a distinction between their function (kitchen at left and living room at right). It shows that the subdivision process could be properly done manually at the designing phase of the models, but since in practice it is not often the case, here we will investigate criteria on which automatic approaches can rely to make and control the process.
Figure 5. Subdivision mainly based on geometric criteria. (a) A corridor space. (b) Its subdivision in two subspaces based on its length size.

\subsection{Geometry-driven criteria}

A first subdivision approach is to purely rely on the geometric properties of the space to process. Typically, parameters such as length, surface area or volume can be used to control with precision where a space cell should be geometrically subdivided. This enables the definition of rules of type: cut the cell( $s$ ) of size s or volume $v$ in half, along and perpendicularly to its longest side. An example is provided in Fig. 5, where a subdivision similar to what is shown in the IndoorGML standard example (see Fig. 4) is presented.

The length of the corridor is represented by the variable $d$. If we decide to subdivide the space into two parts that will have a length equal to $d / 2$, simple computational geometry operations can allow us to perform the cutting. Here, two identical and overlapping faces will be needed in order to have closed volumes in both of their sides (see the red faces in Fig. 5(b)). Other geometric constraints, such as convexity criteria could be used as well. It is even more relevant in this context as indoor navigation algorithms often take the centroid of the cells as nodes of their graph. But the issues related to the graph will be discussed later in this paper.

The advantage of such approach lies in the fact that it does not need to rely on any other information but the geometry. Indeed, there is no need to explicitly know that a space is a corridor for example, the criteria holding independently of the type of cell. But such semantic criteria can also be used to target specific types of cells in the cellular space.

Geometric information are not always intuitive enough to reflect the hierarchical structure of indoor environment properly. Indeed, only considering the space in itself and ignoring its surrounding spaces (topological relationships) may lead to incoherent subdivision. By relying on models of type IFC or CityGML (LoD4), we can take advantage of all the geometric, semantic but also topological information, in order to set the subdivision on more relevant properties of the spaces.

\subsection{Topology-semantic-driven criteria}

Relying on the topological information of the 3D indoor models allows to set sharper rules for the subdivision. Here the topological information corresponds to the spatial relationships linking the cells. This enables the definition of rules of type: cut the space where there is no contact with a wall. But here also, it does not exclude the use of semantic and/or geometry. By setting 


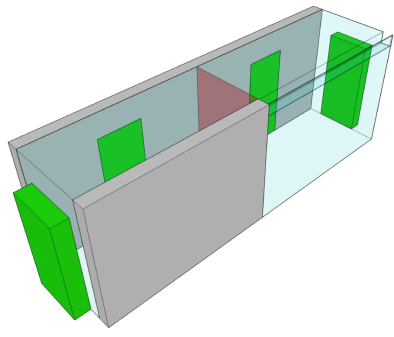

(a)

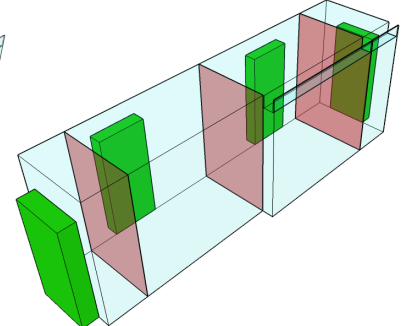

(b)
Figure 6. Subdivision mainly based on topological criteria. (a) Sub-spacing considering the relations to the walls (result similar to the subdivision in Fig. 5). (b) Sub-spacing considering the relations to the doors.

specific rules on the space cells, based on their surrounding features, we can define criteria that are spatially more meaningful. We illustrate some examples of such process in Fig. 6.

Typically, the subdivision previously obtained in Fig. 5 where only geometric criteria were used can also be reproduced, as shown in Fig. 6(a). But there is a considerable difference in the sense that here the sub-spacing is done considering the part of the corridor that is bounded by walls. This implicitly indicates which part of it is eventually connected to another space, therefore it makes it relevant to separate both parts of the corridor's cell space. The topological relationships of the latter have been used then, in addition to the semantic to identify the walls. Fig. 6(b) shows another example where it is decided to cut the corridor at intervals where there is no door, so that each subspace contains one single door. Once again the leading criteria is topological relationships translated by the detection of places where the corridor is related to doors.

\subsection{Navigation-driven criteria}

Purely geometric or topological criteria are not able to solve all complex cases of providing a proper subdivision. However, one can guess that the their combination, in addition to geometric properties can help to build stronger rules that makes a clear sense for indoor navigation. Following the example of the corridor, Fig. 7 shows a specific criteria motivated by the definition of space cells in front of each door around the corridor that can host at least one pedestrian. The size of the cells are set to $1.5 \times 1 \times 2.5 \mathrm{~m}$, to make them spacious enough. This leads to the creation of intersecting cells as illustrated by the red volumes in Fig. 7(a). But the IndoorGML standard only allows common boundary between cells and not volume intersection between them (Lee et al., 2014). Therefore, the merging of the cells can be performed until no intersection exists any more (see Fig. 7(b)). We then end up with three subspaces for the corridor: one for each couple of doors at both extremities of the cell, and one central cell (Fig. 7(c)). Such subdivision may have some convenience in situations where doors' closeness between neighbouring rooms is be involved (e.g. emergency situations).

We have been showing sub-spacing examples just on the cell space of the corridor so far. If we get back to the case of the bathroom of Fig. 3, we can see the impact of excluding the occupied space, as discussed in section 3. Figure 8 reflects the results of a criteria based on the possibility for a pedestrian to walk in the space of the bathroom. To do so, we first need to identify the walkable surface in the corresponding cell. This means that the

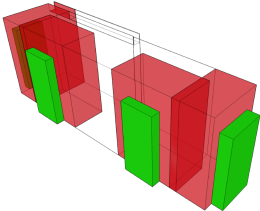

(a)

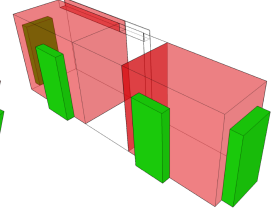

(b)

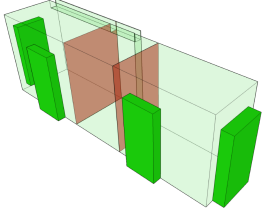

(c)
Figure 7. Subdivision based on geometric, topological and semantic criteria. (a) Result similar to the subdivision in Fig. 5 . (b) Special sub-spacing criteria at door-corridor relations. (c) Merging of intersecting subspaces. (d) Final subdivision of the corridor.

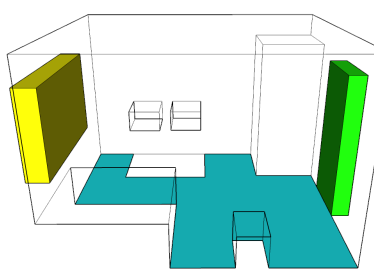

(a)

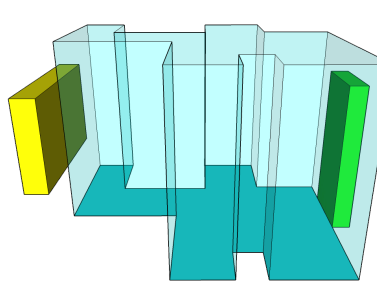

(c)

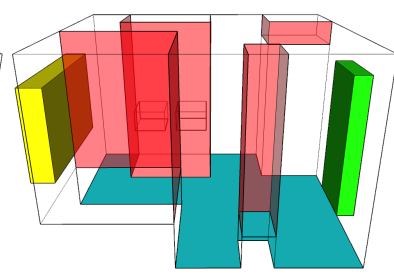

(b)

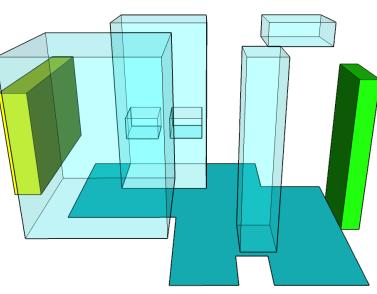

(d)
Figure 8. Subdivision based on walk possibility criteria. (a)

Walkable surface (dark cyan) in the cell space of 3(d). (b)

Cutting faces leading to the targeted sub-spacing. (c) Walkable subspace. (d) Non-walkable subspaces.

occupied spaces need to be excluded first because the navigation will only hold on free spaces. Therefore, using the space as illustrated in Fig. 3(d) is more relevant than the one of Fig. 3(a). The walking possibility can be semantically related to the ground surface, thus the slab of the storey. So using the topological relationships between the cell and the slab, the walkable surface can be identified as shown in Fig. 8(a). The next step consists in identifying where to cut the space cell in order to isolate different categories of subspaces. This can be done by relying on the boundaries of the occupied subspaces, leading to the result shown in Fig. 8(b). Finally, Fig. 8(c) and (d) show the resulting space cells corresponding the the walkable space unit (c) and non-walkable one (d).

In summary, the automatic sub-spacing will always be driven by either geometric, topological or semantic information, or their proper combination in order to generate more specific subspaces. It is the case when generating subspaces dedicated to specific navigation constraints, as discussed above. Since the purpose of IndoorGML is to ultimately provide a navigation graph after defining the cellular space properly, we will explore in the next section the issues related to the extraction of a navigation graph in the context of a subdivided cellular space. 


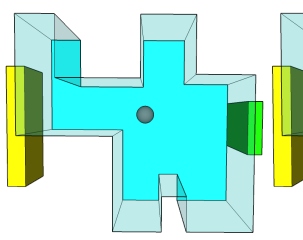

(a)

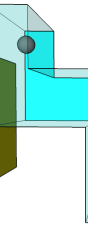

(b)
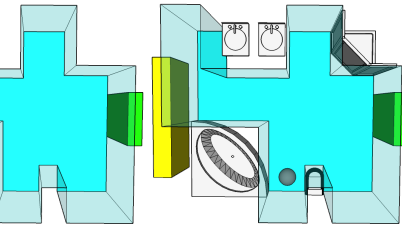

(c)

Figure 9. Example of different positions for a node of the same cell (a) Central position of the node. (b) Node placed at the closest corner to the window. (c) Node placed between two furnishing elements.

\section{EXTRACTING NAVIGATION GRAPH IN A SUBDIVIDED CELLULAR SPACE}

As mentioned earlier, IndoorGML handles the network representation of the cellular space through the NRG. This is motivated by the need of an explicit description of the spatial relationships between the cells. Those links correspond essentially to the adjacency and connectivity relationships among indoor objects. The whole concept used relies on the Poincaré Duality as a theoretical background (Lee et al., 2014). Thus IndoorGML handles a primal and a dual space. The latter is characterized by a multilayered graph structure composed by the graphs of all the layers represented in the model and their inter-layer connections (Kang and $\mathrm{Li}, 2017)$. In each space layer, the corresponding cells are abstracted by a node and its links to the neighbouring cells by an edge. Here we will explore the attribution of those nodes and edges as allowed by the IndoorGML framework, and we will discuss its execution after the sub-spacing process.

\subsection{Node attribution to cells}

A node simply corresponds to a point in IndoorGML. In the standard it is referred to as a State and no real constraints are defined for the practical generation of such node. Indeed the standard does not seem to provide restriction concerning the placement of such node, but for the targeted applications, such constraints may be necessary. It is implicitly assumed that a node representing a space should be at least contained in that space. This is because the nodes of a navigation graph is often associated to a position in the space where the navigating agent can stay. Thus, similar constraint will apply for the IndoorGML framework as illustrated in Fig. 4, even though it is not clearly specified in the standard.

It is a common practice in indoor navigation to use the centroid of a space as its node. This is also the case of the example illustrated in Fig. 4. However, such practice strongly relies on the assumption of convex space units, because the centroid may not be located inside the space otherwise. Such assumption is made easy by the consideration of the space cell as they are described in Fig. 2(b), meaning without consideration of their contained elements. The direct consequence is that the nodes generated with such simplified spaces are likely to end up at a position where the user cannot reach. This could be of considerable impact in critical navigation processes such as emergency situation for example. Therefore, considering the occupancy of the cells is definitely critical to a precise navigation system.

However, as it can be seen in Fig. 3 and 8, the consideration of the occupied spaces leads to highly non-convex space cells (Diakité and Zlatanova, 2016). Therefore, more advanced techniques should be considered for the generation of nodes for such cells.

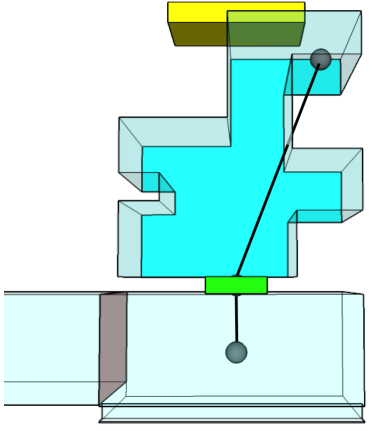

(a)

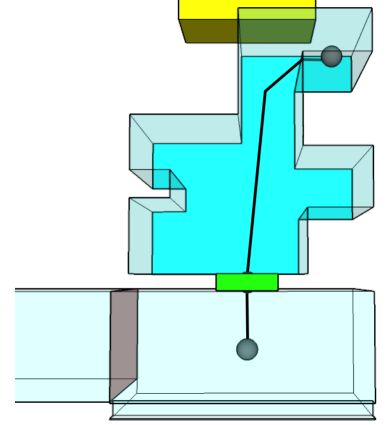

(b)
Figure 10. Edges for the navigation graph. (a)The edge crosses the navigable cell and lay on a non-navigable one. (b) The non-navigable cell is bypassed through the use of multiple edges.

Furthermore, we can rely once again on the geometry, topology and semantic information to put more smartness in the position of the node. Figure 9 shows node placement on the walkable cell in Fig. 8(c). In Fig. 9(a), the node is placed at a central position. Despite the fact that the cell is non-convex, it can happen that its centroid, or the center of its bounding still lies inside it. Such configuration is useful to navigate the moving agent at a central point of the space. Figure 9(b) illustrates a case where, for example, the user targets a walkable corner of the room, near the window. The topological relationship of the cell with the window and the slab can then be used to identify which side of it is the most suitable to place the node. Similar information can also be used in the example of Fig. 9 where the semantic information, along with the topology will allow to identify the two furnishing elements between which the node shall be placed, for a user targeting either of those objects. Hence, the good point in not restricting the nodes to the central points of the cells is the possibility to elaborate several configurations at convenience.

Although IndoorGML rely on the NRG paradigm, it is noteworthy to mention that in some cases, a logical graph can be used to define a path for a user. Indeed, the sequence of navigable cells only could be provided without need to define nodes, their links and xyz coordinates. Thus there is still a door opened to non-geometric approaches.

\subsection{Links between the nodes in the graph}

After placing the nodes in the cells, the next step consists in linking them according to their spatial relationships. They are represented by edges and the IndoorGML standards refers to them as Transition entities. A Transition is then defined as an edge representing the adjacency and connectivity of related cells and always connects two cells (Lee et al., 2014). From that definition, it seems that only the direct links between two cells through one edge is considered. In the case where the graph is restricted to the representation of the spatial links this is not an issue. However, as soon as the graph reflects paths that are supposed to be followed by navigating agents, problems may arise from the fact that the edge can cross other cells supposedly excluded from the real route. An illustration is provided in Fig. 10(a) where a direct link from the node of the room's cell to the door and the corridor crosses the cells in a non walk-able area.

Such issues may be solved in two ways within IndoorGML:

- either by using curves instead of straight edges 
- or by further subdividing the cells into convex subspaces.

Indeed, it is mentioned in the standard that the curve primitive from the GML standard is used for the geometrical representation of a Transition. This includes the use of the gml:LinearString class that is conceptually a single edge, with intermediate point between the origin and the destination node. That makes it possible to derive curves similar to what is illustrated in Fig. 10(b) and allows to provide graphs faithful to paths that can be followed while respecting the navigation constraints. The intermediate points are not meant to serve as position nodes though, thus they are not considered as State entities in the IndoorGML schema.

A second solution is to push the subdivision further and transform the non-convex navigable cells in sets of convex cells so that the centroids of the latter can be used as intermediate points when needed. Such approach would be computationally more expensive for the subdivision but would enable quicker path extraction.

\section{DISCUSSION}

In this paper we identified and discussed several issues related to the IndoorGML standard:

- the identification of the space free of obstacle: we have shown that navigable space is highly dependent on the indoor furniture. The furnishing elements can be modelled and made available in IFC or CityGML models. Alternatively information about free space can be obtained from measurements (mobile scanners and cameras);

- subdivision of the indoor space to provide more accurate localization and navigation: such subdivision is critical if functional spaces have to be identified and additional nodes need to be included in the NRG. We have demonstrated that meaningful subdivision can be obtained using semantic and topological criteria or combination of them. Geometric criteria are straightforward and therefore commonly used, but they are less intuitive for human use;

- node placement in a space: it is commonly assumed that the placement of a node is of no importance. However, in indoor navigation systems relying on paradigms such as NRG, a node represents a point with defined spatial coordinates. In this respect, it becomes critical where the point is placed. We have shown and discussed several cases of different placement possibilities;

- creating the NRG: linking the spaces to create a path for navigation has not been discussed here. IndoorGML prescribes that this should be done on the basis of adjacency, connectivity and accessibility between spaces. Such information can be derived again on semantic and topological information. In this paper we have concentrated on the links between the nodes. Clearly, directly linking the points representing the nodes may create wrong paths. This issue can be resolved either by a further subdivision to allow only convex cells or by using curves to represent the links.

This paper comes as recommendations for IndoorGML to include elaborations on the above mentioned issues. Further investigations and experiments are needed to estimate the size and shape of the cells obtained with a specific space subdivision procedure.
Methods for automatic NRG creation with respect to the user profile need to be investigated as well.

Furthermore, we have not discussed aggregation of spaces in this paper. Indeed, in many cases a more general view of the building may be needed instead of a sub-spacing, e.g. only a floor or a part of a building (Sithole and Zlatanova, 2016). Therefore other rules will be needed to guide the process of uniting spaces and this will also be part of a future work.

\section{ACKNOWLEDGEMENTS}

This research is funded by the NWO/STW M4S project Smart 3D indoor models to support crisis management in large public buildings (www.SIMs3D.net).

\section{REFERENCES}

Becker, T., Nagel, C. and Kolbe, T. H., 2009. A multilayered space-event model for navigation in indoor spaces. In: $3 \mathrm{D} \mathrm{geo-}$ information sciences, Springer, pp. 61-77.

Building SMART International, 2013. Industry Foundation Classes (IFC), IFC4.

Diakité, A. A. and Zlatanova, S., 2016. Extraction of the 3d free space from building model for indoor navigation. ISPRS Ann. Photogramm. Remote Sens. Spatial Inf. Sci. 4(2/W1), pp. 241248.

Haklay, M. and Weber, P., 2008. Openstreetmap: User-generated street maps. IEEE Pervasive Computing 7(4), pp. 12-18.

Hwang, J.-R., Kang, H.-Y. and Choi, J.-w., 2012. Development of an editor and a viewer for indoorgml. In: Proceedings of the Fourth ACM SIGSPATIAL International Workshop on Indoor Spatial Awareness, ACM, pp. 37-40.

Iida, H., Hiroi, K., Kaji, K. and Kawaguchi, N., 2015. A proposal of indoorgml extended data model for pedestrian-oriented voice navigation system. In: Proceedings of the Seventh ACM SIGSPATIAL International Workshop on Indoor Spatial Awareness, ACM, p. 2.

Jung, H. and Lee, J., 2015. Indoor subspacing to implement indoorgml for indoor navigation. ISPRS-International Archives of the Photogrammetry, Remote Sensing and Spatial Information Sciences 1, pp. 25-27.

Kang, H.-K. and Li, K.-J., 2017. A standard indoor spatial data modeloge indoorgml and implementation approaches. ISPRS International Journal of Geo-Information 6(4), pp. 116.

Khan, A. A., Donaubauer, A. and Kolbe, T. H., 2014. A multistep transformation process for automatically generating indoor routing graphs from existing semantic $3 \mathrm{~d}$ building models. In: Proceedings of the 9th 3D GeoInfo Conference.

Kim, J.-S., Yoo, S.-J. and Li, K.-J., 2014. Integrating indoorgml and citygml for indoor space. In: International Symposium on Web and Wireless Geographical Information Systems, Springer, pp. 184-196.

Kim, M. and Lee, J., 2015. Developing a method to generate indoorgml data from the omni-directional image. ISPRSInternational Archives of the Photogrammetry, Remote Sensing and Spatial Information Sciences 1, pp. 17-19.

Kim, Y., Kang, H. and Lee, J., 2013. Development of indoor spatial data model using citygml ade. ISPRS-International Archives of the Photogrammetry, Remote Sensing and Spatial Information Sciences 1(2), pp. 41-45. 
Klepeis, N. E., Nelson, W. C., Ott, W. R., Robinson, J. P., Tsang, A. M., Switzer, P., Behar, J. V., Hern, S. C. and Engelmann, W. H., 2001. The national human activity pattern survey (nhaps): a resource for assessing exposure to environmental pollutants. Journal of Exposure Science and Environmental Epidemiology 11(3), pp. 231.

Krūminaite, M. and Zlatanova, S., 2014. Indoor space subdivision for indoor navigation. In: Proceedings of the Sixth ACM SIGSPATIAL International Workshop on Indoor Spatial Awareness, ACM, pp. 25-31.

Lee, J., Li, K., Zlatanova, S., Kolbe, T., Nagel, C. and Becker, T., 2014. OGC® IndoorGML. Available from: http://www.opengeospatial.org/standards/indoorgml.

Li, K.-J., 2016. Indoorgml-a standard for indoor spatial modeling. International Archives of the Photogrammetry, Remote Sensing \& Spatial Information Sciences.

Li, K.-J. and Lee, J., 2010. Indoor spatial awareness initiative and standard for indoor spatial data. In: Proceedings of IROS 2010 Workshop on Standardization for Service Robot, Vol. 18.

Mirvahabi, S. and Abbaspour, R. A., 2015. Automatic extraction of indoorgml core model from openstreetmap. The International Archives of Photogrammetry, Remote Sensing and Spatial Information Sciences 40(1), pp. 459.

Nations, U., 2014. World urbanization prospects: The 2014 revision, highlights. department of economic and social affairs. Population Division, United Nations.

Open Geospatial Consortium, 2012. City Geography Markup Language (CityGML) Encoding Standard, version 2.0.0.

Ryoo, H.-G., Kim, T. and Li, K.-J., 2015. Comparison between two ogc standards for indoor space: Citygml and indoorgml. In: Proceedings of the Seventh ACM SIGSPATIAL International Workshop on Indoor Spatial Awareness, ACM, p. 1.

Ryu, H.-G., Kim, T. and Li, K.-J., 2014. Indoor navigation map for visually impaired people. In: Proceedings of the Sixth ACM SIGSPATIAL International Workshop on Indoor Spatial Awareness, ACM, pp. 32-35.

Sithole, G. and Zlatanova, S., 2016. Position location, place and area: An indoor perspective. ISPRS Ann. Photogramm. Remote Sens. Spat. Inf. Sci 4, pp. 89-96.

Zlatanova, S., Li, K.-J., Lemmen, C. and van Oosterom, P., 2016a. Indoor abstract spaces: Linking indoorgml and ladm. In: Proceedings of the 5th International FIG Workshop on $3 D$ Cadastres, International Federation of Surveyors (FIG).

Zlatanova, S., Liu, L. and Sithole, G., 2013. A conceptual framework of space subdivision for indoor navigation. In: Proceedings of the Fifth ACM SIGSPATIAL International Workshop on Indoor Spatial Awareness, ACM, pp. 37-41.

Zlatanova, S., Liu, L., Sithole, G., Zhao, J., Mortari, F., Liu, L., Sithole, G., Zhao, J. and Mortari, F., 2014. Space subdivision for indoor applications. Delft University of Technology, OTB Research Institute for the Built Environment.

Zlatanova, S., Van Oosterom, P., Lee, J., Li, K.-j. and Lemmen, C., 2016b. Ladm and indoorgml for support of indoor space identification. ISPRS Annals of Photogrammetry, Remote Sensing and Spatial Information Sciences pp. 257-263. 